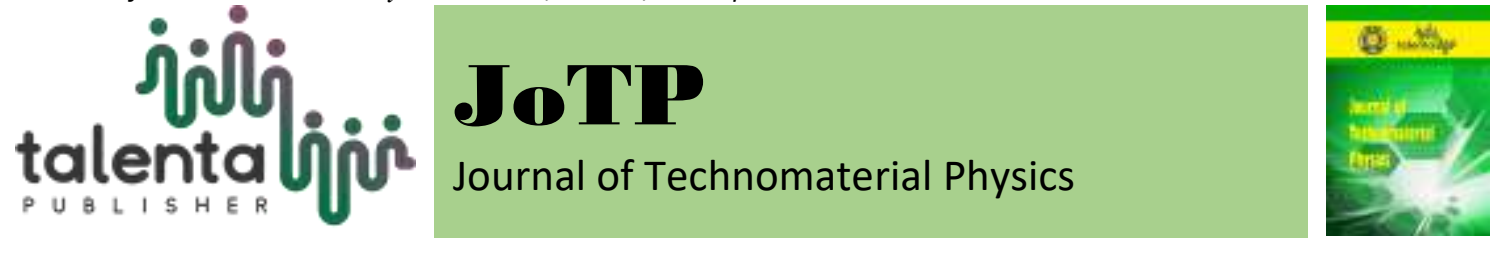

\title{
The Gravitomagnetic Effects in Rapidly Rotating Neutron Stars: A Theoretical Study
}

\author{
Atsnaita Yasrina 1 and Nugroho Adi Pramono ${ }^{2}$ \\ ${ }^{1,2}$ Departmen of Physiscs, Faculty of Matematics and Natural Sciences, Universitas Negeri Malang, \\ Indonesia, 20155
}

\begin{abstract}
Electromagnetic measurements of a general relativistic gravitomagnetic effect can be done within the conductor embedded in a rotating gravitational object's spacetime. Neutron stars are rotating gravitational object that have strong magnetic field. The gravitomagnetic effect in a neutron star can be determined from the distribution density in the conductor. Neutron star is assumed as a conductor and it rotates rapidly. The distribution density inside the conductor is obtained from the electromagnetic contravariant tensor and the relativistic rotational speed of the conductor. It has obtained the distribution density inside the conductor for the rapidly rotating neutron star. The results are compared to the slowly rotating neutron star which depends on the angular velocity and the gravitational field.
\end{abstract}

Key Words: gravitomagnetic, charge distribution, neutron star, rapidly rotating.

Received 14 July 2020 | Revised [28 July 2020] | Accepted [27 August 2020]

\section{Introduction}

Many astrophysicists have studied the magnetic field and the magnetism of neutron stars [1]. The discovery of neutron star as a pulsar has a strong magnetic field. Pulsar is a rapidly rotating neutron star [2]. The magnetic field of pulsar can be seen from the emission of the electromagnetic waves in the form of radio waves and X-rays [3]. Radio pulsars have magnetic field of $B \sim 10^{8} \mathrm{G}-10^{13} \mathrm{G}$ [2]. X-rays pulsars have magnetic field of $B>10^{12} \mathrm{G}$ [1]. The maximum magnetic field of an X-ray pulsar is $B \sim 10^{15} \mathrm{G}$ [4]. In general, the maximum magnetic field of a neutron star is $B \sim 10^{18} \mathrm{G}[5]$.

The magnetic field of a neutron star undergoes a change or magnetic evolution [4-8]. The magnitude of the neutron star's magnetic field can be reduced to the order of $\sim 10^{-4}$ than before [9]. The cause of this change in magnetic field is the accretion process [6-14]. Theoretically, the magnetic field dynamics equation has been formulated for the slowly rotating

*Corresponding author at: Jl. Semarang, No.05, Malang, Jawa Timur Indonesia, 20155

E-mail address: Atsnaita.yasrina.fmipa@um.ac.id 
neutron star [15] and the rapidly rotating one [16]. In the formulation of the magnetic field dynamics equation, the neutron star can be assumed as a conducting object.

Apart from that as a conductor, neutron stars have very strong gravity. The energy of gravity is $E_{\text {grav }} \sim G M^{2} / R \sim 5 \times 10^{53} \mathrm{erg} \sim 0,2 M c^{2}$, with the gravitational acceleration on its surface is $g \sim G M / R^{2} \sim 2 \times 10^{14} \mathrm{~cm} \mathrm{~s}^{-2}[1]$. As conductors and gravitational objects, neutron stars have gravitmagnetic field. There have been many studies on general relativistic electromagnetic effects arising from the gravitomagnetic field in conductor without the external magnetic field [17]. There have also been studied for the same case but with the external magnetic field [18]. The conductor is the slowly rotating gravitational object. The results obtained indicate the form of charge density in the conductor with the external magnetic field. This charge density is affected by the angular velocity $\Omega$, gravitational field. The metric used in this study is a metric for slowly rotating gravitational object [18], which can be used for the slowly rotating neutron stars. The rapidly rotating neutron stars are also conductors and gravitational objects. Therefore, it is necessary to know the general relativistic electromagnetic effects arising from the gravitomagnetic field of this rapidly rotating neutron star, and how it is compared to the slowly rotating neutron star.

\section{Methods}

The method used in this research is analytic theoretical-mathematical analysis. In order to determine the gravitomagnetic effect, it can be seen from the charge distribution in the conductor $\left(\rho_{0}\right)$. The metric used is the rapidly rotating neutron star metric. Metrics are used to formulate the Christoffel symbol $\left(\Gamma_{a \beta}^{\gamma}\right)$, the 4-velocity vector for conductor $\left(u^{\mu}\right)$, and the contravariant component of the electromagnetic tensor $\left(F^{\alpha \beta}\right)$. The 4-velocity vector for conductor $\left(u^{\mu}\right)$ is used to formulate the absolute acceleration of the conductor $\left(w_{a}\right)$. The 4velocity vector for conductor $\left(u^{\mu}\right)$ and the absolute acceleration of the conductor $\left(w_{a}\right)$ are used to formulate the relative rotational velocity of the conductor $\left(A_{\beta \alpha}\right)$. The charge distribution in the conductor $\left(\rho_{0}\right)$ is formulated from the contravariant component of the electromagnetic tensor $\left(F^{\alpha \beta}\right)$ and the relative rotational speed of the conductor $\left(A_{\beta \alpha}\right)$. The research procedure is shown in Figure (1). 


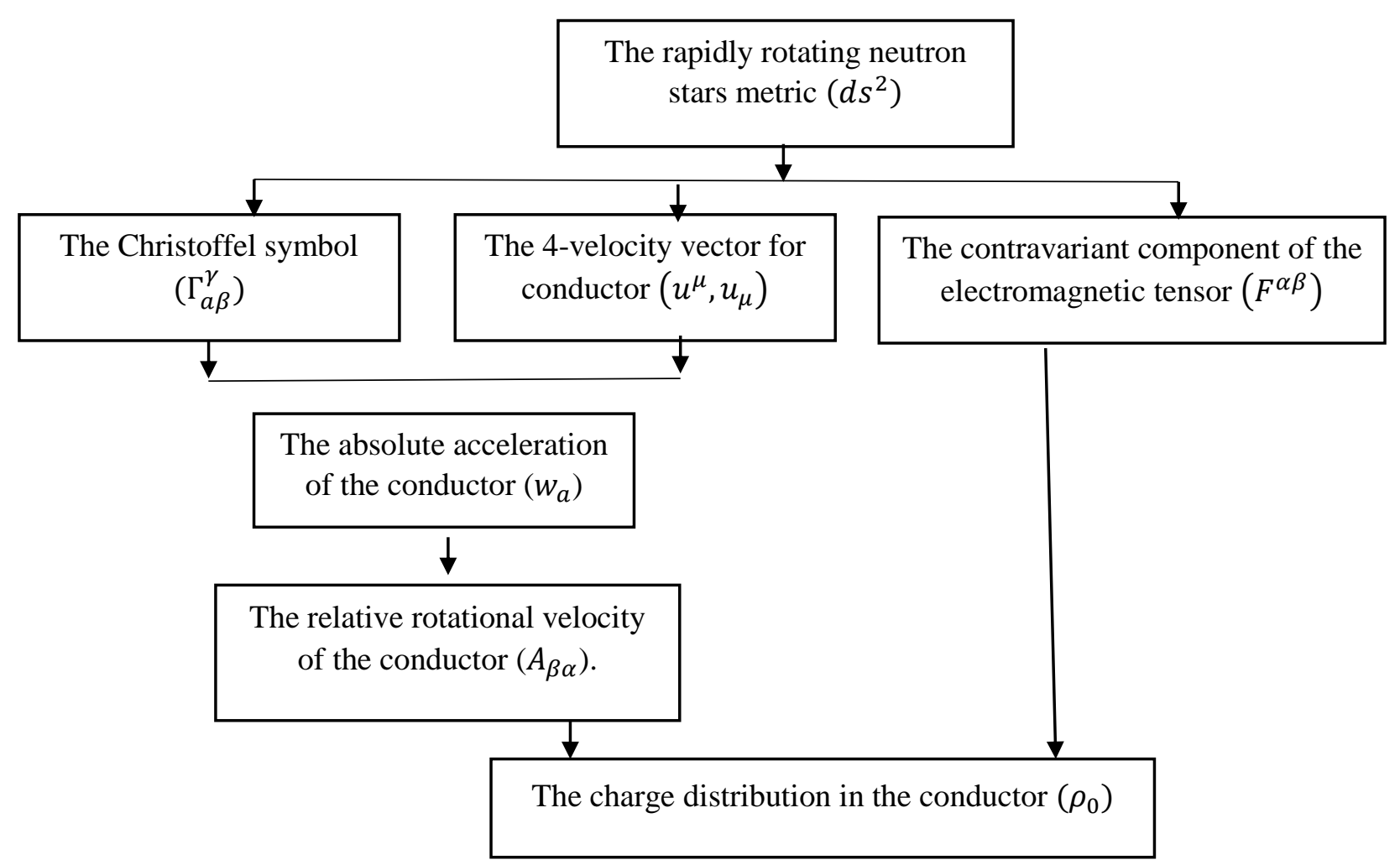

Figure 1. The research procedure

\subsection{Metric, Christtofel Symbol, 4-velocity Vector for The Conductor}

Equation (1) is a rapidly rotating neutron star metrics

$$
d s^{2}=-e^{2 \phi} d t^{2}+e^{2 \lambda} r^{2} \sin ^{2} \theta(d \varphi-\omega d t)^{2}+e^{2 \alpha}\left(d r^{2}+r^{2} d \theta^{2}\right)
$$

With the functions of $\phi, \lambda, a$, and $\omega$ depends on the $r$ and $\theta$ [19]. The metric component is

$$
g_{\mu \nu}=\left[\begin{array}{cccc}
-p & 0 & 0 & -q \\
0 & k & 0 & 0 \\
0 & 0 & l & 0 \\
-q & 0 & 0 & h
\end{array}\right]
$$

and the inverse is

$$
g^{\mu \nu}=\left[\begin{array}{cccc}
-f^{-1} & 0 & 0 & -X \\
0 & k^{-1} & 0 & 0 \\
0 & 0 & l^{-1} & 0 \\
-X & 0 & 0 & -Y
\end{array}\right]
$$

with 


$$
\begin{gathered}
f(r, \theta)=e^{2 \phi} ; h(r, \theta)=e^{2 \lambda} r^{2} \sin ^{2} \theta ; k(r, \theta)=e^{2 \alpha} ; l(r, \theta)=e^{2 \alpha} r^{2} ; \\
p(r, \theta)=\left(f-h \omega^{2}\right) ; q(r, \theta)=h \omega ; X(r, \theta)=f^{-1} \omega ; \\
Y(r, \theta)=\frac{\left(f-h \omega^{2}\right)}{f h} ; Z(r, \theta)=\sqrt{-g}=e^{\phi+2 \alpha+\lambda} r^{2} \sin \theta .
\end{gathered}
$$

Metric equation (1) is used to formulate the Cristoffel symbol, including

$$
\begin{gathered}
\Gamma_{00}^{0}=1 ; \Gamma_{03}^{0}=0 ; \Gamma_{01}^{0}=\frac{1}{Z} Z_{, r} ; \Gamma_{02}^{0}=\frac{1}{Z} Z_{, \theta} ; \Gamma_{33}^{0}=0 ; \\
\Gamma_{13}^{0}=\frac{1}{2}\left[f^{-1} q_{, r}-X h_{, r}\right] ; \Gamma_{23}^{0}=\frac{1}{2}\left[f^{-1} q_{, \theta}-X h_{, \theta}\right] ; \\
\Gamma_{00}^{3}=0 ; \Gamma_{03}^{3}=0 ; \Gamma_{10}^{3}=\frac{1}{2}\left[X p_{, r}+Y q_{, r}\right] ; \\
\Gamma_{13}^{3}=\frac{1}{2}\left[X q_{, r}-Y h_{, r}\right] ; \Gamma_{23}^{3}=\frac{1}{2}\left[X q_{, \theta}+Y h_{, \theta}\right] ; \\
\Gamma_{33}^{3}=0 ; \Gamma_{30}^{3}=0 ; \Gamma_{20}^{3}=\frac{1}{2}\left[X p_{, \theta}+Y q_{, \theta}\right]
\end{gathered}
$$

Metric equation (1) is also used to formulate the 4-velocity vector for conductor, as follows [16]

$$
u^{\mu}=\frac{1}{\sqrt{p}}(1,0,0, \sqrt{y})
$$

with the contravariance component is

$$
u_{\mu}=\left(\left(-\sqrt{p}-q \sqrt{\frac{Y}{p}}\right), 0,0,\left(-\frac{q}{\sqrt{p}}+h \sqrt{Y}\right)\right)
$$

\subsection{The Contravariant tensor of the Electromagnetic Field}

The relationship between the electric 4-vector field $E^{\alpha}$ and magnetic $B^{\alpha}$ is shown by the electromegnetic field tensor. The covariance component of the electromagnetic tensor is

$$
F_{\alpha \beta} \equiv 2 u_{[\alpha} E_{\beta]}+\eta_{\alpha \beta \gamma \delta} u^{\gamma} B^{\delta}
$$

where $u^{\mu}$ is the 4-velocity vector, and $\eta_{\alpha \beta \gamma \delta}$ is the pseudo-tensorial. The equation (8) produces a covariant tensor component of the electromagnetic field of rapidly rotating neutron star [20] 


$$
\begin{aligned}
& F_{00}=F_{11}=F_{22}=F_{33}=0, \\
& F_{01}=-F_{10}=-\Phi E_{r}-\Psi \omega B^{\theta}, \\
& F_{02}=-F_{20}=-\Phi E_{\theta}+\Psi \omega B^{r}, \\
& F_{03}=-F_{30}=-\Phi E_{\varphi}, \\
& F_{12}=-F_{21}=\Psi B^{\varphi}, \\
& F_{13}=-F_{31}=-\Psi B^{\theta}, \\
& F_{23}=-F_{32}=\Psi B^{r},
\end{aligned}
$$

and the contravariant tensor of the electromagnetic field are

$$
\begin{aligned}
& F^{00}=F^{11}=F^{22}=F^{33}=0, \\
& F^{01}=-F^{10}=E^{r} / \Phi, \\
& F^{02}=-F^{20}=E^{\theta} / \Phi, \\
& F^{03}=-F^{30}=E^{\phi} / \Phi, \\
& F^{12}=-F^{21}=B_{\varphi} / \Psi, \\
& F^{13}=-F^{31}=-\left(\Phi B_{\theta}+\Psi \omega E^{r}\right) / \Psi \Phi, \\
& F^{23}=-F^{32}=\left(\Phi B_{r}-\Psi \omega E^{\theta}\right) / \Psi \Phi,
\end{aligned}
$$

with

$$
\Psi(r, \theta)=\left(e^{2 \alpha+\lambda} r^{2} \sin \theta\right) / \sqrt{1-V^{2}},
$$

and

$$
\Phi(r, \theta)=e^{\phi} / \sqrt{1-V^{2}} .
$$

\subsection{Relativistic Rotation Speed in The Conductor}

The absolute acceleration of the conductor $\left(w_{a}\right)$ satisfies the equation (13)

$$
w_{a}=u_{a ; \beta} u^{\beta}=\left(u_{a, \beta}-\Gamma_{a \beta}^{\gamma} u_{\gamma}\right) u^{\beta}
$$

Equations (5), (6), and (7) are substituted into the equation (13), obtained as follow

$$
\begin{aligned}
w_{0}= & \left(-\sqrt{p}-q \sqrt{\frac{y}{p}}\right) \frac{1}{\sqrt{p}} \\
w_{1}= & -\frac{1}{Z} Z_{, r}\left(-\sqrt{p}-q \sqrt{\frac{Y}{p}}\right) \frac{1}{\sqrt{p}}-\frac{1}{2}\left[X p_{, r}+Y q_{, r}\right]\left(-\sqrt{p}-q \sqrt{\frac{Y}{p}}\right) \sqrt{\frac{Y}{p}} \\
& -\frac{1}{2}\left[f^{-1} q_{, r}-X h_{, r}\right]\left(-\sqrt{p}-q \sqrt{\frac{Y}{p}}\right) \sqrt{\frac{Y}{p}}-\frac{1}{2}\left[X q_{, r}-Y h_{, r}\right]\left(-\frac{q}{\sqrt{p}}+h \sqrt{Y}\right) \sqrt{\frac{Y}{p}} \\
w_{2}= & -\frac{1}{Z} Z_{, \theta}\left(-\sqrt{p}-q \sqrt{\frac{Y}{p}}\right) \frac{1}{\sqrt{p}}-\frac{1}{2}\left[X q_{, \theta}+Y h_{, \theta}\right]\left(-\frac{q}{\sqrt{p}}+h \sqrt{Y}\right) \frac{1}{\sqrt{p}} \\
& -\frac{1}{2}\left[f^{-1} q_{, \theta}-X h_{, \theta}\right]\left(-\sqrt{p}-q \sqrt{\frac{Y}{p}}\right) \sqrt{\frac{Y}{p}}-\frac{1}{2}\left[X p_{, \theta}+Y q_{, \theta}\right]\left(-\frac{q}{\sqrt{p}}+h \sqrt{Y}\right) \sqrt{\frac{Y}{p}}
\end{aligned}
$$


$w_{3}=0$

The absolute acceleration of the conductor in the slowly rotating neutron star is only the component $w_{1} \neq 0$ [21]. Meanwhile, for the rapidly rotating neutron star component is $w_{o}, w_{1}, w_{2} \neq 0$.

The relative rotational speed of the conductor $\left(A_{\beta \alpha}\right)$ satifies the equation

$$
A_{\beta \alpha}=u_{\alpha, \beta}+u_{\left[{ }_{\beta} w_{a}\right]}
$$

Equations (6), (7), and (13a-13.d) are substituted into the equation (15), obtained as follows

$$
\begin{aligned}
& A_{00}=A_{11}=A_{22}=A_{33}=0, \\
& A_{01}=-A_{10}=\left(\sqrt{p}+q \sqrt{\frac{Y}{p}}\right)_{, r}-\frac{1}{2}\left(\sqrt{p}+q \sqrt{\frac{Y}{p}}\right) w_{1}, \\
& A_{02}=-A_{20}=\left(\sqrt{p}+q \sqrt{\frac{Y}{p}}\right)_{, \theta}-\frac{1}{2}\left(\sqrt{p}+q \sqrt{\frac{Y}{p}}\right) w_{2}, \\
& A_{03}=-A_{30}=0, \\
& A_{12}=-A_{21}=0, \\
& A_{13}=-A_{31}=\left(-\frac{q}{\sqrt{p}}+h \sqrt{Y}\right)_{, r}-\frac{1}{2}\left(-\frac{q}{\sqrt{p}}+h \sqrt{Y}\right) w_{1}, \\
& A_{23}=-A_{32}=\left(-\frac{q}{\sqrt{p}}+h \sqrt{Y}\right)_{, \theta}-\frac{1}{2}\left(-\frac{q}{\sqrt{p}}+h \sqrt{Y}\right) w_{2} .
\end{aligned}
$$

\subsection{The Charge Density inside The Conductor}

The charge distribution inside the conductor in general relativistics is

$$
\begin{aligned}
& \rho_{0}=\frac{\epsilon \mu R_{H}}{c} j^{2}+\frac{1}{4 \pi}\left\{\left(\frac{\epsilon}{\lambda} j^{\alpha}\right)_{; \alpha}+\left[\epsilon^{2} \mu R_{H}\left(\frac{1}{\lambda} j^{2}+\Lambda^{-\frac{1}{2}} j^{\nu} \nabla_{\nu}^{\perp}\left(\Lambda^{\frac{1}{2}} \mu_{e}\right)\right) u^{\alpha}\right]_{; \alpha}-\epsilon R_{g g} A_{\alpha \beta} w^{\alpha} j^{\beta}+\right. \\
& g^{\alpha \beta}\left(\epsilon R_{g g} j^{v} A_{\alpha \nu}\right)_{; \beta}-\frac{\epsilon}{\lambda} w^{\alpha} j_{\alpha}-\epsilon w^{a} \Lambda^{-\frac{1}{2}} \nabla_{\alpha}^{\perp}\left(\Lambda^{\frac{1}{2}} \mu_{e}\right)+g^{\alpha \beta}\left(\epsilon \Lambda^{-\frac{1}{2}} \nabla_{\alpha}^{\perp}\left(\Lambda^{\frac{1}{2}} \mu_{e}\right)\right)_{; \beta}+ \\
& \left.H^{\alpha \beta}\left[A_{\alpha \beta}+\epsilon \mu R_{H} w_{a} j_{\beta}+\left(\epsilon \mu R_{H} j_{\alpha}\right)_{, \beta}\right]\right\}
\end{aligned}
$$


where $j^{a}$ is the conduction current, $\epsilon$ and $\mu$ is the parameter for the conductor, $\mu_{e}$ is the electrochemical potential per unit charge, $R_{g g}=2 m c / n e^{2}$ is the parameter for a conductor called as galvano-gravitomagnetic one, $n$ is the consentration of conduction electrons, $\lambda$ is the electrical conductivity, $R_{H}$ is the Hall constant, and $\nabla_{a}^{\perp}$ denotes the spatial part of the covariant derivative [18][21].

The charge density $\rho_{0}$ in a conductor that has no conductive current $j=0$ but is embedded in the external magnetic field $\mathbf{B}$ is [18][21]

$$
\rho_{0}=\frac{1}{4 \pi}\left\{\epsilon A w^{2}-\left(\epsilon A w^{\alpha}\right)_{; \alpha}+F^{\alpha \beta} A_{\beta \alpha}\right\} \text {. }
$$

Equation (18) has two contributions, the first is due to the absolute acceleration $w_{a}$ and the second is due to the relativistic rotation rate of the conductor $A_{\beta a}$, and $A$ is the parameter for the conductor [18][21]. In this case, it does not consider the redistribution of charge arising from the absolute acceleration of the conductor because it does not depend on the characteristics of electromagnetic field so that the charge density inside the conductor is assumed to be $[18,21]$.

$$
\rho_{0}=-\frac{1}{2 \pi}\left(F^{a \beta} A_{a \beta}\right)
$$

Equation (10.a-10.g) and (16.a-16.g) are substituted into the equation (19), obtained as follows

$$
\begin{aligned}
& \rho_{0}=-\frac{1}{2 \pi}\left[F^{01} A_{01}+F^{02} A_{02}+F^{13} A_{13}+F^{23} A_{23}\right] \\
& =-\frac{1}{2 \pi}\left[\left(\frac{E^{r}}{\Phi}\right)\left(\left(\sqrt{p}+q \sqrt{\frac{Y}{p}}\right)_{, r}-\frac{1}{2}\left(\sqrt{p}+q \sqrt{\frac{Y}{p}}\right) w_{1}\right)+\left(\frac{E^{\theta}}{\Phi}\right)\left(\left(\sqrt{p}+q \sqrt{\frac{Y}{p}}\right)_{, \theta}-\right.\right. \\
& \left.\frac{1}{2}\left(\sqrt{p}+q \sqrt{\frac{Y}{p}}\right) w_{2}\right)+\left(-\frac{\left(\Phi B_{\theta}+\Psi \omega E^{r}\right)}{\Psi \Phi}\right)\left(\left(-\frac{q}{\sqrt{p}}+h \sqrt{Y}\right)_{, r}-\frac{1}{2}\left(-\frac{q}{\sqrt{p}}+h \sqrt{Y}\right) w_{1}\right)- \\
& \left.\left(\frac{\left(\Phi B_{\theta}+\Psi \omega E^{r}\right)}{\Psi \Phi}\right)+\left(\frac{\left(\Phi B_{r}-\Psi \omega E^{\theta}\right)}{\Psi \Phi}\right)\left(\left(-\frac{q}{\sqrt{p}}+h \sqrt{Y}\right)_{, \theta}-\frac{1}{2}\left(-\frac{q}{\sqrt{p}}+h \sqrt{Y}\right) w_{2}\right)\right]
\end{aligned}
$$

If it is compared to the slowly rotating neutron star shown in the equation (20), then the equation (19) has more complex shape. For the slow rotation, the charge density in the conductor depends on the angular velocity $\Omega$ and the gravitational field [18]. For the slowly rotating general relativistic effect can be amplified by the interaction between the gravitomagnetic field and the magnetic field. In this study, it uses a test or trial, which is an Earth's gravitational field using a conductor embedded in an external magnetic field [18]. The conclusion is that there is a galvano-gravitomagnetic effect (interaction between the gravitomagnetic field and the electric current). This new effect occurs when the current carrying conductor is placed in the gravitomagnetic field and the conduction electrons move inside the conductor [18]. The equation (19) contains an angular velocity $\Omega$ (i.e. in a function of $V$ ) and a gravitational field. However, because the equation (19) has more complex shape. So, in order to determine the gravitomagnetic effect in the future, a certain measurement method is needed. In 
this article, it only presents the theoretical study of a rapidly rotating spacetime as the comparison for the slowly rotating spacetime.

\section{Conclusion}

The electromagnetic measurements of the general relativistic gravitomagnetic effects can be done inside the conductor embedded in a rotating gravitational object's spacetime. The deep gravitomagnetic effect can be discovered from the distribution density in the conductor. In this case, the conductor embedded in the rotating gravitational object's spacetime is the rapidly rotating neuron star. The distribution density in the conductor for the rapidly rotating neuron star is shown in the equation (19). The obtained results are compared to the slowly rotating neutron stars. If it is compared to the slowly rotating neutron star shown in the equation (20), then the equation (19) has more complex shape. However, the distribution density in the conductor still depends on the angular velocity and the gravitational field which the same as the slowly rotating that indicates the galvano-gravitomagnetic effect. In the slow rotation studies, it has been proven using test or trial of the Earth's gravitational field using the embedded conductor in the external magnetic field. For the future study, it is desirable that certain measurement methods are required for the rapidly rotating neutron stars.

\section{REFERENCES}

[1] P. Haensel, A. Y. Potekhin, and D. G. Yakovlev, Neutron stars 1: Equation of state and structure, vol. 326, no. 1. 2007.

[2] M. Camezind, Compact Objects in Astrophysics. 2007.

[3] A. Reisenegger, "Evolution of neutron star magnetic fields," Astrophys. Space Sci., vol. 140, no. 1, pp. 39-47, 2003, doi: 10.1007/BF00643526.

[4] A. Reisenegger, "Magnetic field evolution in neutron stars," Astron. Nachrichten, vol. 328 , no. 10, pp. 1173-1177, 2007, doi: 10.1002/asna.200710848.

[5] A. Y. Potekhin, "The physics of neutron stars," Physics-Uspekhi, vol. 53, no. 12, pp. 1235-1256, 2010, doi: 10.3367/ufne.0180.201012c.1279.

[6] K. Konar and B. Dipankar, "Magnetic field evolution of accreting neutron stars," Mon.Not.R.Astron.Soc, vol. 284, p. 1997, 1997.

[7] D. Bhattacharya, "Evolution of neutron star magnetic fields," J.Astrophys. Astr, vol. 23, pp. 67-72, 2002, doi: 10.1007/BF00643526.

[8] A. Cumming, E. Zweibel, and L. Bildsten, "Magnetic Screening in Accreting Neutron Stars," Astrophys. J., vol. 557, no. 2, pp. 958-966, Aug. 2001, doi: 10.1086/321658.

[9] C. M. Zhang, "Accretion induced crust screening for the magnetic field decay of neutron stars," Astron. Astrophys., vol. 330, no. 1, pp. 195-200, 1998.

[10] U. Anzer and G. Borner, "Accretion by Neutron Stars: Accretion Disk and Rotating Magnetic Field," Astron. Astrophys., vol. 83, pp. 133-139, 1980.

[11] A. Melatos and E. S. Phinney, "Hydromagnetic structure of a neutron star accreting at its polar caps," Publ. Astron. Soc. Aust., vol. 18, no. 4, pp. 421-430, 2001, doi: 10.1071/AS01056. 
[12] S. Konar and A. Choudhuri, "Diamagnetic Screening of the Magnetic Field of an Accreting Neutron Star," 34th COSPAR Sci. Assem., vol. 34, no. March, pp. 1-9, 2002.

[13] R. V. E. Lovelace, M. M. Romanova, and G. S. Bisnovatyi-Kogan, "Screening of the Magnetic Field of Disk Accreting Stars," Astrophys. J., vol. 625, no. 2, pp. 957-965, 2005, doi: $10.1086 / 429532$.

[14] W. C. G. Ho, "Evolution of a buried magnetic field in the central compact object neutron stars," Mon. Not. R. Astron. Soc., vol. 414, no. 3, pp. 2567-2575, 2011, doi: 10.1111/j.1365-2966.2011.18576.x.

[15] L. Rezzolla and B. J. Ahmedov, "Electromagnetic fields in the exterior of an oscillating relativistic star - I. General expressions and application to a rotating magnetic dipole," Mon. Not. R. Astron. Soc., vol. 352, no. 4, pp. 1161-1179, 2004, doi: 10.1111/j.13652966.2004.08006.x.

[16] A. Yasrina and D. Andra, "The magnetic field dynamics equation of the accreting and rapidly rotating neutron star in the ZAMO (Zero Angular Momentum Observers) frame," J. Phys. Conf. Ser., vol. 1231, no. 1, 2019, doi: 10.1088/1742-6596/1231/1/012030.

[17] I. Ciufolini and J. A. Wheeler, Gravitation and Inertia. Princeton Univ. Press, New Jersey: Princeton Univ. Press, 1995.

[18] B. J. Ahmedov and M. Karim, "Gravitomagnetic Effects in a Conductor in an Applied Magnetic Field," Ann. Phys, vol. 9, pp. 1-5, 2006, [Online]. Available: http://arxiv.org/abs/gr-qc/0608021.

[19] Gregory B Cook, S. S. L, and At. Saul, "Rapidly Rotating Neutron Stars in General Relativity: Realistic Equations of State,” Astrophys. J., vol. 424, pp. 823-845, 1994.

[20] A. Yasrina, "Tensor Kontravarian Medan Elektromagnetik Bintang Neutron Yang Berotasi Cepat Diukur Oleh Pengamat Zamo (Zero Angular Momentum Observers)," J. Fis., vol. 5, no. 1, p. 79791, 2015, doi: 10.15294/jf.v5i1.7372.

[21] B.J. Ahmedov, "General relativistic galvano-gravitomagnetic effect in current carrying conductors B.J. Ahmedov 1," Phys. Lett. A, vol. 256, pp. 1-10, 1999. 